\title{
Resistencia adhesiva dentina- resina utilizando tres sistemas adhesivos a diferentes tiempos de aplicación. Estudio in vitro
}

\section{Resin dentin bond's resistance using three adhesive systems at different application times. Study in vitro}

\begin{abstract}
Resumen
Objetivo. Evaluar la resistencia adhesiva dentina-resina (RADR) en adhesivos Optibond FL (OFL), Adper Single Bond 2 (ASB2) y Single Bond Universal (SBU) en tres tiempos diferentes de aplicación. Métodos. Estudio experimental in vitro, se empleó nueve molares humanas obteniéndose, 135 especímenes de $1 \mathrm{~mm}^{2}$ aproximadamente conformando nueve grupos de estudio de 15 especímenes para cada grupo. La RADR se midió a través del test de microtensión a una fuerza de tracción con una aceleración de $0,5 \mathrm{~mm} / \mathrm{min}$ hasta la fractura. El contraste de hipótesis de diferencia se realizó utilizando el test de ANOVA de un factor y comparaciones múltiples de Tukey, ambos a un nivel de confianza del 95\%. Resultados. A los 10 segundos, se hallaron diferencias significativas entre los sistemas adhesivos OFL $(25,78 \pm 5,98 \mathrm{MPa})$ vs ASB2 (de 14,02 $\pm 3,06 \mathrm{MPa}), \mathrm{p}<0,001$, así como entre OFL vs $\mathrm{SBU}(14,36 \pm 4,69 \mathrm{MPa})$ con $\mathrm{p}<0,001$. A los 20 segundos, entre OFL $(34,37 \pm 6,03 \mathrm{MPa})$ y ASB2 $(16,33 \pm 3,02 \mathrm{MPa}), \mathrm{p}<0,001$, así como entre OFL y SBU $(19,44 \pm 5,98 \mathrm{MPa})$ con $\mathrm{p}<0,001$. A los 30 segundos también se encontraron las mismas diferencias entre OFL $(37,6 \pm 11,93 \mathrm{MPa})$ y ASB2 $(16,62 \pm 1,81 \mathrm{MPa})$, $\mathrm{p}<0,001$, así como entre OFL y SBU $(23,56 \pm 7,3 \mathrm{MPa}) \mathrm{p}<0,001$. Conclusiones. $O p-$ tibond FL presentó mayor RADR frente a ASB2 y SBU en los tres tiempos; entre ASB2 y SBU a 10 s no se encontró diferencia, a los 20 s y 30 s SBU presentó mayor RADR. En los tres grupos hubo aumento significativo de la RADR entre los 10 y 30 segundos.
\end{abstract}

Palabras clave: Dentina; Adhesión dental; Adhesivos (fuente: DeCS BIREME).

\section{Artículo Original}

Ketty Amelia Arquíñego Garay ${ }^{1, a}$, Armando Del Castillo Ayquipa ${ }^{1, b}$, Rómel Watanabe Velásquez ${ }^{1, c}$

${ }^{1}$ Universidad Nacional Mayor de San Marcos, Facultad de Odontología. Lima, Perú.

a Cirujano Dentista.

${ }^{\mathrm{b}}$ Maestro en Odontología.

c Maestro en Estomatología.

\section{Correspondencia:}

Ketty Amelia Arquíñego Garay

Correo electrónico: kettyaag@hotmail.com

Jr. Julián Alarcón 993. San Martin de Porres Lima 31, Perú.

\section{Coautores:}

Armando Del Castillo Ayquipa

armandodel228@yahoo.es

Rómel Watanabe Velásquez

rwatanabev@unmsm.edu.pe

\section{Editora:}

Dahiana Alfaro Carballido

Universidad de San Martín de Porres, Perú.

Conflicto de intereses: los autores declaran no tener conflictos de interés.

Fuente de financiamiento: recursos propios de los investigadores.

Recibido: 13/01/19

Aceptado: $11 / 03 / 19$

Publicado: 05/06/19 
a confidence level of $95 \%$. Results. After 10 seconds, significant differences were found between the OFL adhesive systems $(25.78 \pm 5.98 \mathrm{MPa})$ vs ASB2 (from $14.02 \pm 3.06$ $\mathrm{MPa})$ at $\mathrm{p}<0.001$, as well as between OFL vs $\mathrm{SBU}(14.36 \pm 4.69 \mathrm{MPa})$ with $\mathrm{p}<0.001$. At 20 seconds, between OFL $(34.37 \pm 6.03 \mathrm{MPa})$ and ASB2 $(16.33 \pm 3.02 \mathrm{MPa})$ at level $\mathrm{p}<0.001$, as well as between OFL and SBU $(19.44 \pm 5.98 \mathrm{MPa})$ with $\mathrm{p}<0.001$. After 30 seconds, the same differences were found between OFL $(37.6 \pm 11.93 \mathrm{MPa})$ and ASB2 $(16.62 \pm 1.81 \mathrm{MPa})$ at $\mathrm{p}<0.001$, as well as between OFL and SBU $(23.56) \pm 7.3 \mathrm{MPa})$ with $\mathrm{p}<0.001$. Conclusions. OFL presented greater DRBR compared to ASB2 and SBU in the three rubs; between ASB2 and SBU rubbing 10 s no difference was found, rubbing $20 \mathrm{~s}$ and $30 \mathrm{~s}$ SBU presented higher DRBR. In all three groups there was a significant increase in DRBR between 10 and 30 seconds.

Keywords: Dentin; Dental bonding; Adhesives (source: MeSH NLM).

\section{Introducción}

En la práctica clínica odontológica, uno de los materiales mayormente utilizado para tratar la pérdida de tejido dentario por diferentes causas, es sin duda la resina compuesta. Esta puede ser utilizada de forma directa, semi indirecta o indirecta, aplicando protocolos estrictos y delicados para lograr el éxito de la restauración, así como el uso de agentes de unión a los tejidos dentarios como los sistemas adhesivos. A pesar de la estabilidad adhesiva lograda sobre el esmalte dental, estudios respecto a la adhesión en sustrato dentinario revelan que los mecanismos adhesivos todavía son sensibles, impredecibles e inestables ${ }^{1}$. Más aún cuando se trata de una dentina profunda donde hay presencia de mayor humedad, por lo que la interfaz dentina-resina continúa siendo el área más débil de las restauraciones ${ }^{2}$.

Para garantizar una correcta interfaz dentina-resina, la forma de aplicar el adhesivo es de vital importancia. Como mencionan Reis et al. ${ }^{3}$, si el adhesivo se aplica frotando la superficie se incrementa la fuerza adhesiva, esto se debería a un mayor tiempo de permanencia activa del adhesivo sobre dentina antes del fotocurado, existiendo mayor penetración del mismo en el enmallado colágeno, liberando a la vez su solvente ${ }^{4}$. Este frotamiento está indicado estrictamente en dentina e incluso los fabricantes frecuentemente lo recomiendan ${ }^{5}$ para mejorar los niveles de resistencia en dentina. Una de las clasificaciones con base científica de los sistemas adhesivos está basado en el uso o no del ácido fosfórico y en el número de pasos durante su aplicación, resultando sistemas adhesivos llamados Etch and Rinse (grabar y lavar) de tres y dos pasos, y adhesivos Self Etch (autograbado) de dos y un paso ${ }^{6,7}$. La necesidad de mejorar la resistencia adhesiva, perfeccionamiento de la técnica, disminución de la filtración marginal, entre otros factores, justifican la necesidad de crear nuevos sistemas adhesivos que incorporan ventajas frente a los anteriores. Estos llevan por nombre adhesivos multimodales o universales ${ }^{8}$. Los sistemas adhesivos Etch and Rinse llamados también convencionales, mediante el grabado ácido eliminan el barrillo dentinario logrando la hibridación a través de la interdigitación entre el adhesivo y las fibras colágenas, en su mayoría de tipo I ${ }^{6,9}$. Los adhesivos Etch and Rinse de tres pasos, incluyen también tres pasos para su aplicación, donde el primero es considerado al proceso de acondicionamiento con ácido fosfórico ${ }^{6}$; el segundo, la aplicación del "primer" que se presenta en monofrasco y contiene monómeros hidrófilos que promueven la re-expansión del colágeno y facilita su penetración en la dentina húmeda; dentro de estos encontramos el HEMA (hidroxi-etil-metacrilato), está disuelto en un solvente que puede ser alcohol, acetona o agua ${ }^{6}$, cuya función es desplazar el agua de la superficie de la dentina, presenta también monómeros hidrófugos que permiten su unión con el bonding ${ }^{6}$; el tercer paso corresponde a la colocación del adhesivo propiamente dicho llamado "bond" o bonding, que contiene una resina hidrófuga como es el BIS-GMA, y una pequeña cantidad de monómeros hidrófilos ${ }^{6}$. Cuando el "primer" y bond se presenta en un solo frasco, corresponde a un Etch and Rinse de dos pasos ${ }^{10}$. Los sistemas adhesivos Etch and Rinse tienen el inconveniente del manejo adecuado del protocolo de aplicación, teniendo en cuenta el riesgo de sobre humedecer o resecar la dentina después del lavado del ácido fosfórico, degradando las fibras colágenas y presentando mayor posibilidad de sensibilidad posoperatoria ${ }^{11}$. Los sistemas adhesivos Self Etch no requieren de grabado ácido, por lo que se le llama de autograbado ${ }^{6}$. Al igual que los sistemas adhesivos Etch and Rinse, pueden clasificarse en función al número de pasos según su aplicación y a la presentación separada o junta del "primer" acídico y bond, existiendo adhesivos Self Etch de dos y un paso respectivamente ${ }^{10}$. Los Self Etch de dos pasos presentan en un frasco monómeros acídicos hidrófilos que simultáneamente graban e imprimen al sustrato dental, permaneciendo el barro dentinario y la hidroxiapatita disuelta en la capa híbrida que es de menor espesor ${ }^{6,10} \mathrm{y}$ en otro frasco un agente hidrófugo para su posterior polimerización ${ }^{10}$. Los adhesivos Self Etch de un paso mezclan el grabado ácido, imprimación y adhesión en un solo frasco, todo en uno 10,11. Los adhesivos multimodales o universales son una nueva categoría de sistemas adhesivos que tienen la particularidad de ser empleados tanto por la técnica convencional de grabado ácido total, técnica autograbado o por la técnica de grabado ácido selectivo a nivel de esmalte ${ }^{12,13}$, se presentan en un solo frasco. Una de las innovaciones sobre estos adhesivos es la incorporación de una molécula bifuncional Vinil Silano que permite la unión química de sustratos cerámicos como resinosos 12 y monómeros funcionales que se adhieren químicamente al calcio de la hidroxiapatita como el Metacriloxiddecilfosfato dibidrogenado (10-MDP) ${ }^{13}$. De este modo 
los sistemas adhesivos universales brindan una retención química fuerte, además, de la retención micromecánica; esta última lograda también con los dos grandes grupos de adhesivos, Etch and Rinse y Self Etch. El inconveniente de realizar el grabado ácido a través del ácido fosfórico en dentina y someterla a un proceso ácido menor a $1(\mathrm{pH})$, conduce a la liberación de unas enzimas colagenolíticas producidas por los odontoblastos durante la dentinogénesis, permaneciendo desde ese entonces, fosilizadas en la matriz dentinaria, llamadas metaloproteinasas y catepsinas responsables de la destrucción paulatina de la matriz colágena de la capa híbrida ${ }^{14}$. Por otro lado, está demostrado que los sistemas adhesivos son permeables, ya que permite el paso de fluidos a través del adhesivo polimerizado en dentina, ello también afectaría longitudinalmente la estabilidad hidrolítica de la interfaz dentina-adhesivo (resina), más aun si se está próxima a la pulpa donde el fluido dentinario es mayor y la posibilidad de hidrólisis es en menor tiempo ${ }^{15}$. Se entiende como hidrólisis a un proceso químico donde hay ruptura de enlaces covalentes de las moléculas que conforman un polímero, por la adición de agua a los enlaces del tipo éster ${ }^{16}$.

El propósito principal del estudio fue evaluar la resistencia adhesiva dentina-resina de tres sistemas adhesivos: Optibond FL (Etch and Rinse de tres pasos), Adper Single Bond 2 (Etch and Rinse de dos pasos) y Single Bond Universal (con su posibilidad de autograbado) en forma de aplicación activa, frotación en tiempos diferentes (10 s, 20 s y $30 \mathrm{~s}$ ).

\section{Métodos}

La presente investigación es de tipo experimental in vitro.

Selección, preparación de dientes y aplicación del adhesivo. Se seleccionaron nueve terceros molares inferiores humanos recientemente extraídos en consultorios privados, sin rasgos clínicos de caries dental o algún tipo de restauración; fueron desinfectados durante $24 \mathrm{~h}$ en una solución de timol al 0,1\% y almacenados a temperatura ambiente en un recipiente de vidrio con suero fisiológico para evitar su deshidratación, máximo por 24 horas que duró el tiempo de recolección. La recolección de los molares y realización del estudio no presentó riesgo alguno, físico o mental para las personas, por lo que se contó con la aprobación del Comité Institucional de Ética en Investigación del Instituto de Medicina Tropical "Daniel Alcides Carrión” de la Universidad Nacional Mayor de San Marcos.

Los nueve molares fueron divididos en nueve grupos correspondiendo un molar por cada grupo, en relación a cada sistema adhesivo, según el tiempo a aplicar el adhesivo. Las raíces de cada molar fueron sumergidas en un cubo de bisacryl Acrytemp, hasta $2 \mathrm{~mm}$ antes del margen gingival, para posteriormente realizar un corte transversal eliminando el esmalte coronal, mediante la máquina de corte tipo Isomet bajo continua corriente de agua sin necesidad de realizar un pulido posterior; se lavaron las superficies con suero fisiológico por $10 \mathrm{~s}$ con jeringa triple y se secaron con papel tisú. Para cada sistema adhesivo (Etch and Rinse de tres pasos: OptiBond FL, Etch and Rinse de dos pasos: Adper Single Bond $2 \mathrm{y}$ adhesivo Multimodal: Single Bond Universal) se requirió tres grupos, donde se aplicó el adhesivo variando el tiempo de aplicación activa de 10 s, 20 s y 30 s (frotación continua).

Para los sistemas adhesivos OptiBond FL y Adper Single Bond 2 se realizó el grabado con ácido fosfórico al $37 \%$ con la técnica de grabado total, lavándose y secándose con papel tisú, luego se aplicaron los sistemas adhesivos de la siguiente manera: GRUPO A: Sistema Adhesivo Etch and Rinse de tres pasos OptiBond FL (Kerr), aplicando el "primer" activamente en forma de frotación continua durante 10 s. GRUPO B: Sistema Adhesivo Etch and Rinse de tres pasos OptiBond FL (Kerr), aplicando el "primer" activamente en forma de frotación continua durante 20 s. GRUPO C: Sistema Adhesivo Etch and Rinse de tres pasos OptiBond FL (Kerr), aplicando el "primer" activamente en forma de frotación continua durante 30 s. GRUPO D: Sistema Adhesivo Etch and Rinse de dos pasos Adper Single Bond 2 (3M), aplicando activamente en forma de frotación continua durante 10 s. GRUPO E: Sistema Adhesivo Etch and Rinse de dos pasos Adper Single Bond 2 (3M), aplicando activamente en forma de frotación continua durante 20 s. GRUPO F: Sistema Adhesivo Etch and Rinse de dos pasos Adper Single Bond 2 (3M), aplicando activamente en forma de frotación continua durante $30 \mathrm{~s}$.

Para el sistema adhesivo Multimodal Single Bond Universal se lavó la superficie con suero fisiológico por 10 $s$ con jeringa triple, se secó la superficie con papel tisú, luego se aplicó el adhesivo, con su posibilidad de autograbado, en los tres grupos restantes ( $\mathrm{G}, \mathrm{H}$ e I), de la siguiente manera: GRUPO G: Sistema Adhesivo Multimodal Single Bond Universal (3M) sin grabado, aplicando activamente en forma de frotación continua durante 10 s. GRUPO H: Sistema Adhesivo Multimodal Single Bond Universal (3M) sin grabado, aplicando activamente en forma de frotación continua durante $20 \mathrm{~s}$. GRUPO I: Sistema Adhesivo Multimodal Single Bond Universal (3M) sin grabado, aplicando activamente en forma de frotación continua durante $30 \mathrm{~s}$.

En todos los grupos, luego de aplicarse los sistemas adhesivos, se esperó 30 segundos para la volatilización del solvente y luego se procedió a la polimerización, aplicando luz LED con lámpara Elipar DeepCure-L(3M) con intensidad de luz de $1470 \mathrm{~mW} / \mathrm{cm} 2$ durante el tiempo indicado por el fabricante según el adhesivo a usar (Figura 1 A y B).

Proceso restaurador. Para la confección del cubo de resina se construyó, como guía, un aro de silicona transparente Elite Glass (Zhermack) de $5 \mathrm{~mm}$ de altura por 10 $\mathrm{mm}$ de diámetro aproximadamente, que fue colocado sobre la superficie cortada al eliminar el esmalte coronal, dentro de ella se aplicó resina compuesta filtek Z350 XT (3M), con técnica incremental oblicua de $2 \mathrm{~mm}$, cada incremento se polimerizó durante $20 \mathrm{~s}$ con la unidad de 
luz LED modelo Elipar DeepCure-L(3M) con intensidad de luz de $1470 \mathrm{~mW} / \mathrm{cm} 2$ (Figura $1 \mathrm{C}$ y D).

Obtención de especímenes (unidad de estudio). La parte coronal de cada molar se seccionó verticalmente, en sentido frontal y lateral, utilizando la máquina de corte tipo Isomet con irrigación continua, obteniendo 15 barritas o especímenes por cada grupo de estudio, de $1 \mathrm{~mm}^{2}$ aproximadamente, teniendo un total de 135 especímenes (Figura 2).

Aplicación del test de microtensión a los especímenes para la obtención de la resistencia adhesiva. Los especímenes fueron almacenados en agua destilada por 24 horas, trascurrido ese tiempo se llevó al test de microtensión con el Microtensile Tester (BISCO ${ }^{\circ}$ ), para ello se pegó cada barrita al aparato con cianocrilato y se sometió a una fuerza de tensión con una aceleración de 0,5 $\mathrm{mm} / \mathrm{min}$ hasta el fallo. Cuando se produjo la fractura, la tracción que ejerció la máquina se detuvo, registrando las medidas obtenidas en tablas diseñadas para el vaciado de la información y conversión posterior a megapascales $(\mathrm{MPa})$, por ser la unidad que mide la fuerza de resistencia adhesiva (Figura 3 ).
Análisis estadístico. El análisis y procesamiento de datos se realizó a través del programa estadístico SPSS versión 21.0. Los valores obtenidos en MPa fueron resumidos con medidas de tendencia central (media y mediana) y dispersión (desviación estándar, coeficiente de variación). La normalidad de los datos fue corroborado por el test de Shapiro-Wilk. El contraste de hipótesis de diferencia se realizó utilizando el test de ANOVA de un factor y comparaciones múltiple de Tukey, ambos a un nivel de confianza del 95\% aceptando un error tipo I del $5 \%$.

\section{Resultados}

Con respecto a la resistencia adhesiva resina-dentina del sistema adhesivo Etch \& Rinse de tres pasos: Optibond FL (Kerr), aplicados activamente con frotación $10 \mathrm{~s}, 20$ s y 30 s se obtuvo $25,78,34,37$ y $37,60 \mathrm{MPa}$ respectivamente. El sistema adhesivo adhesivo Etch $\&$ Rinse de dos pasos: Adper Single Bond 2 (3M) aplicados activamente con frotación $10 \mathrm{~s}$, 20 s y $30 \mathrm{~s}$ se obtuvo 14,02, 16,33 y $16,62 \mathrm{MPa}$ a $10 \mathrm{~s}, 20 \mathrm{~s}$ y $30 \mathrm{~s}$ de frotación respectivamente y con el sistema adhesivo Multimodal:

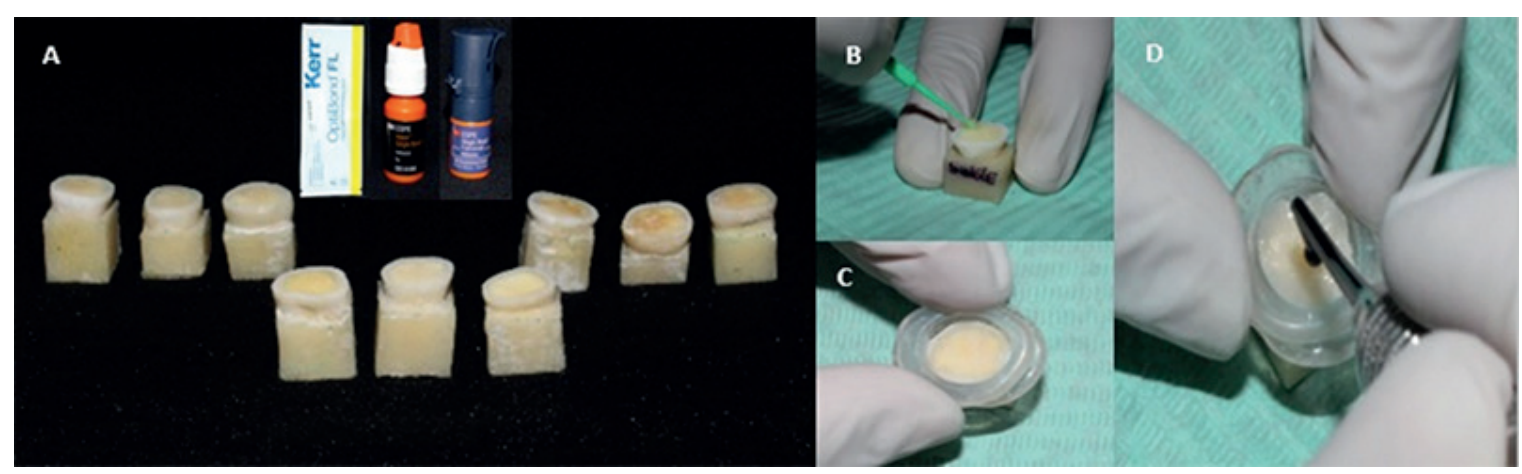

Figura 1. A. Sistemas adhesivos y grupos de estudio. B. Aplicación activa del sistema adhesivo. C. Colocación del aro de silicona transparente. D. Aplicación de la resina compuesta

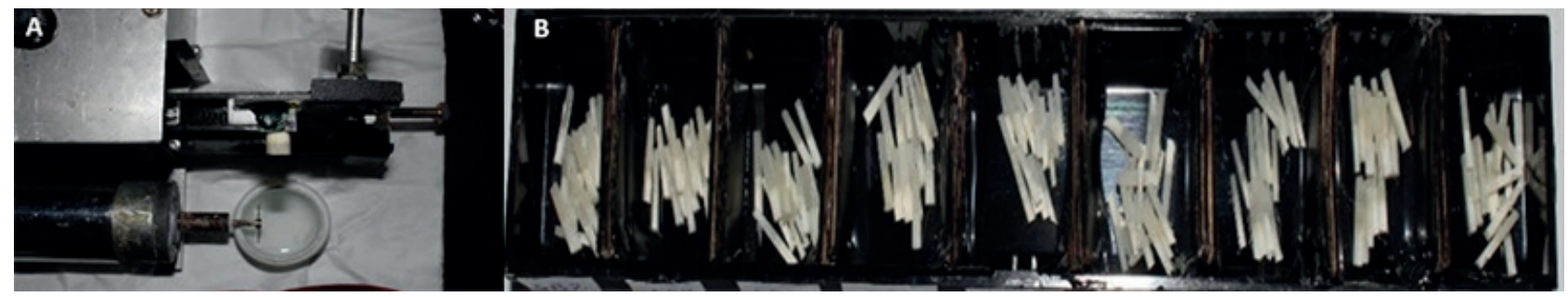

Figura 2. A. Máquina de corte tipo Isomet. B. Quince especímenes por grupo de estudio

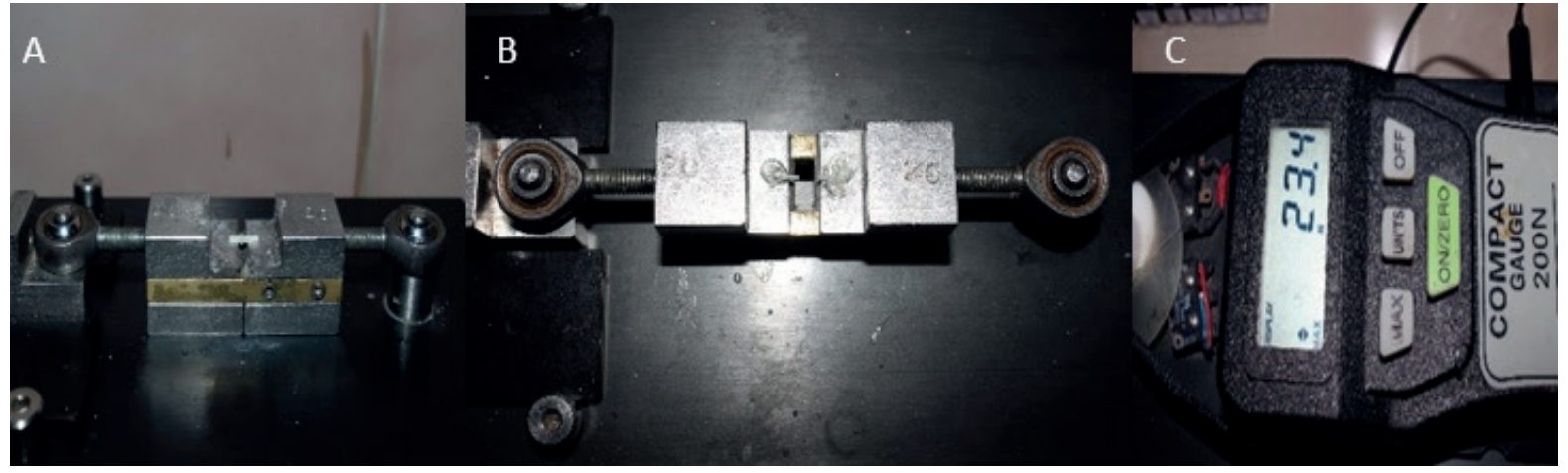

Figura 3. A. Espécimen en Microtensile Tester. B. Fractura. C. Medida de la fuerza que provocó la fractura 
Single Bond Universal (3M) se obtuvo 14,36, 19,44 y $23,56 \mathrm{MPa}$ a $10 \mathrm{~s}, 20 \mathrm{~s}$ y $30 \mathrm{~s}$ de frotación respectivamente (Tablas 1, 2 y 3 ).

En la comparación de la resistencia adhesiva resina-dentina entre los diferentes grupos de estudio según el tiempo de frotación, empleando el mismo adhesivo (prueba de comparaciones múltiples de Tukey), se obtuvo que dentro del grupo del sistema adhesivo Etch $\&$ Rinse de tres pasos: Optibond FL (Kerr), se encontró diferencia estadísticamente significativa entre el grupo de frotación de $10 \mathrm{~s}$ vs $20 \mathrm{~s}$ ( $\mathrm{p}=0,022)$, así como entre $10 \mathrm{~s}$ vs $30 \mathrm{~s}$ $(\mathrm{p}=0,001)$. Dentro del grupo del sistema adhesivo Etch $\&$ Rinse de dos pasos: Adper Single Bond 2 (3M) se encontró diferencia estadísticamente significativa entre el grupo de frotación de $10 \mathrm{~s}$ vs $30 \mathrm{~s}(\mathrm{p}=0,03)$, en los otros grupos las diferencias de resistencia adhesiva no fueron significativas y para el grupo del sistema adhesivo Multimodal: Single Bond Universal (3M) se encontró diferencia estadísticamente significativa solo en los grupos de frotación de $10 s$ vs $30 s(\mathrm{p}<0,001)$ (Tabla 4 y Figura 4).

Tabla 1. Valores descriptivos de resistencia adhesiva dentina-resina (MPa) del sistema adhesivo Etch and Rinse de tres pasos: OptiBond FL (Kerr), aplicando activamente con frotación $10 \mathrm{~s}, 20 \mathrm{~s}$ y $30 \mathrm{~s}$

\begin{tabular}{ccccccccc}
\hline \multirow{2}{*}{ Tiempo } & $\begin{array}{c}\text { Media } \\
\text { (Mpa) }\end{array}$ & DE & LI & LS & Mediana & Mín & Máx & CV \\
\hline $10 \mathrm{~s}$ & 25,78 & 5,98 & 22,47 & 29,09 & 25,16 & 17,37 & 39,15 \\
$20 \mathrm{~s}$ & 34,37 & 6,03 & 31,03 & 37,71 & 36,09 & 20,30 & 43,50 & $17,5 \%$ \\
$30 \mathrm{~s}$ & 37,60 & 11,93 & 31,00 & 44,21 & 34,79 & 24,48 & 67,15 & $31,7 \%$ \\
\hline
\end{tabular}

*Significancia estadística; ${ }^{+}$Basado en el test de ANOVA de un factor. DE: desviación estándar; IC: Intervalo de confianza; CV: Coeficiente de variación

Tabla 2. Valores descriptivos de resistencia adhesiva dentina-resina ( $\mathrm{MPa}$ ) del sistema adhesivo Etch and Rinse de dos pasos: Adper Single Bond 2 (3M), aplicando activamente con frotación 10 s, 20 s y 30 s

\begin{tabular}{|c|c|c|c|c|c|c|c|c|c|}
\hline \multirow{2}{*}{ Tiempo } & \multirow{2}{*}{$\begin{array}{l}\text { Media } \\
\text { (Mpa) }\end{array}$} & \multirow{2}{*}{ DE } & \multicolumn{2}{|c|}{ IC 95\% } & \multirow{2}{*}{ Mediana } & \multirow{2}{*}{ Min } & \multirow{2}{*}{ Máx } & \multirow{2}{*}{$\mathrm{CV}$} & \multirow{2}{*}{ Valor $\mathrm{p}^{\dagger}$} \\
\hline & & & LI & LS & & & & & \\
\hline $10 \mathrm{~s}$ & 14,02 & 3,06 & 12,33 & 15,71 & 13,30 & 8,93 & 19,20 & $21,8 \%$ & \\
\hline $20 \mathrm{~s}$ & 16,33 & 3,02 & 14,66 & 18,01 & 15,81 & 11,61 & 22,04 & $18,5 \%$ & $0,022^{*}$ \\
\hline $30 \mathrm{~s}$ & 16,62 & 1,81 & 15,62 & 17,63 & 16,32 & 14,11 & 19,99 & $10,9 \%$ & \\
\hline
\end{tabular}

*Significancia estadística; ${ }^{+}$Basado en el test de ANOVA de un factor. DE: desviación estándar; IC: Intervalo de confianza; CV: Coeficiente de variación

Tabla 3. Valores descriptivos de resistencia adhesiva dentina-resina (MPa) del sistema adhesivo Multimodal, con su posibilidad de autograbado: Single Bond Universal (3M), aplicando activamente con frotación $10 \mathrm{~s}, 20 \mathrm{~s}$ y $30 \mathrm{~s}$

\begin{tabular}{|c|c|c|c|c|c|c|c|c|c|}
\hline \multirow{2}{*}{ Tiempo } & \multirow{2}{*}{$\begin{array}{c}\text { Media } \\
\text { (Mpa) }\end{array}$} & \multirow{2}{*}{ DE } & \multicolumn{2}{|c|}{ IC 95\% } & \multirow{2}{*}{ Mediana } & \multirow{2}{*}{ Min } & \multirow{2}{*}{ Máx } & \multirow{2}{*}{$\mathrm{CV}$} & \multirow{2}{*}{ Valor $\mathrm{p}^{\dagger}$} \\
\hline & & & LI & LS & & & & & \\
\hline $10 \mathrm{~s}$ & 14,36 & 4,69 & 11,76 & 16,96 & 13,03 & 8,60 & 22,66 & $32,7 \%$ & \\
\hline $20 \mathrm{~s}$ & 19,44 & 5,98 & 16,13 & 22,75 & 19,00 & 9,03 & 29,10 & $30,8 \%$ & $0,001^{*}$ \\
\hline $30 \mathrm{~s}$ & 23,56 & 7,30 & 19,51 & 27,60 & 21,45 & 14,04 & 38,59 & $31,0 \%$ & \\
\hline
\end{tabular}

*Significancia estadística; ${ }^{+}$Basado en el test de ANOVA de un factor. DE: desviación estándar; IC: Intervalo de confianza; CV: Coeficiente de variación.

Tabla 4. Comparación de la resistencia adhesiva resina-dentina entre los diferentes grupos de estudio, empleando el mismo adhesivo

\begin{tabular}{|c|c|c|c|c|c|c|}
\hline \multirow{2}{*}{ Adhesivos } & \multirow{2}{*}{ Frotaciones } & \multirow{2}{*}{$\begin{array}{c}\text { Diferencia de } \\
\text { medias (I-J) }\end{array}$} & \multirow{2}{*}{$\begin{array}{c}\text { Error están- } \\
\text { dar }\end{array}$} & \multirow{2}{*}{ Valor $\mathrm{p}^{\dagger}$} & \multicolumn{2}{|c|}{ IC $95 \%$} \\
\hline & & & & & Límite inferior & Límite superior \\
\hline \multirow{3}{*}{ OptiBond FL } & $10 \mathrm{vs} 20 \mathrm{~s}$ & $-8,5900$ & 3,08714 & $0,022^{*}$ & $-16,0902$ & $-1,0898$ \\
\hline & $10 \mathrm{vs} 30 \mathrm{~s}$ & $-11,8233$ & 3,08714 & $0,001^{*}$ & $-19,3235$ & $-4,3231$ \\
\hline & 20 vs $30 \mathrm{~s}$ & $-3,2333$ & 3,08714 & 0,552 & $-10,7335$ & 4,2669 \\
\hline \multirow{3}{*}{$\begin{array}{c}\text { Adper Single Bond } \\
2(3 M)\end{array}$} & $10 \mathrm{vs} 20 \mathrm{~s}$ & $-2,31267$ & ,98329 & 0,060 & $-4,7016$ & ,0762 \\
\hline & 10 vs $30 \mathrm{~s}$ & $-2,59933^{*}$ & ,98329 & $0,030^{*}$ & $-4,9882$ &,- 2104 \\
\hline & $20 \mathrm{vs} 30 \mathrm{~s}$ &,- 28667 & ,98329 & 0,954 & $-2,6756$ & 2,1022 \\
\hline \multirow{3}{*}{$\begin{array}{l}\text { Single Bond Uni- } \\
\text { versal }(3 \mathrm{M})\end{array}$} & $10 \mathrm{vs} 20 \mathrm{~s}$ & $-5,07267$ & 2,22190 & 0,069 & $-10,4708$ & 3254 \\
\hline & $10 \mathrm{vs} 30 \mathrm{~s}$ & $-9,19533^{*}$ & 2,22190 & $<0,001^{*}$ & $-14,5934$ & $-3,7972$ \\
\hline & 20 vs $30 \mathrm{~s}$ & $-4,12267$ & 2,22190 & 0,164 & $-9,5208$ & 1,2754 \\
\hline
\end{tabular}




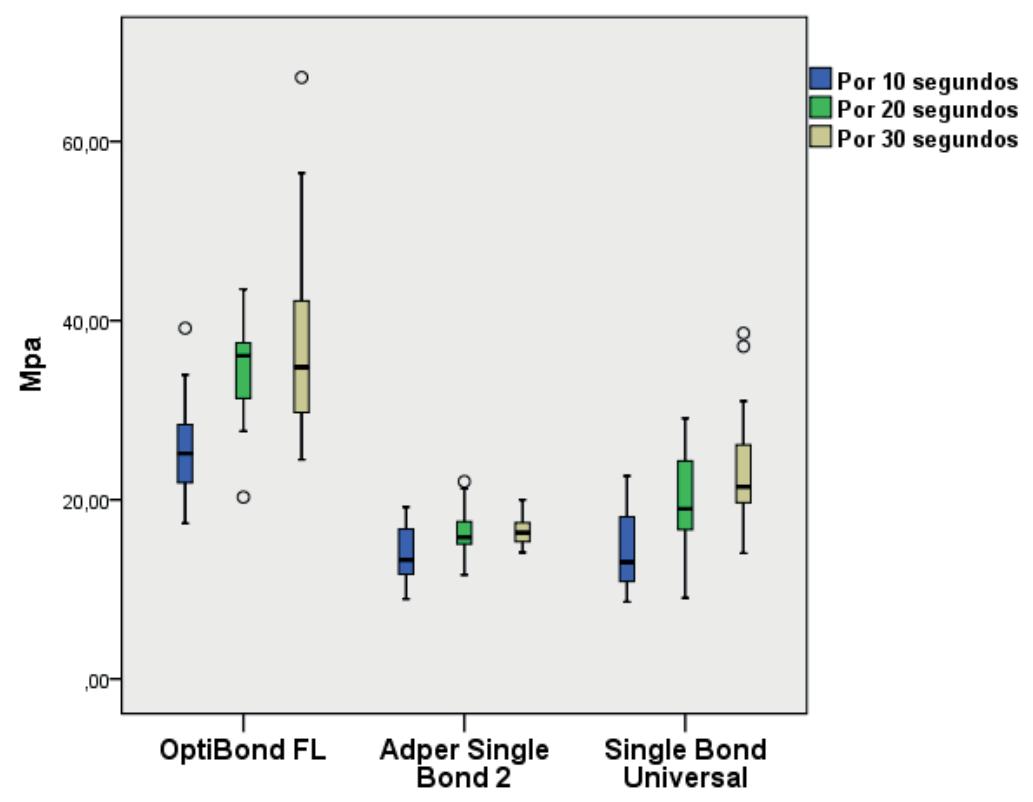

Figura 4. Distribución de valores de resistencia adhesiva en los diferentes grupos de estudio, empleando el mismo adhesivo

En cuanto a la comparación de la resistencia adhesiva dentina-resina entre los diferentes sistemas adhesivos utilizados, dentro de cada grupo de frotación se encontró que en la aplicación de los tres sistemas adhesivos (Optibond FL, Adper Single Bond 2 y Single Bond Universal) con frotación de $10 \mathrm{~s}, 20 \mathrm{~s}$ y $30 \mathrm{~s}$ existen diferencias estadísticamente significativas entre los sistemas adhesivos Optibond FL vs Adper Single Bond 2 ( $\mathrm{p}<0,001)$, así como entre Optibond FL vs Single Bond Universal $(\mathrm{p}<0,001)$ de la misma manera en los tres tiempos de frotación (Tabla 5 y Figura 5).

\section{Discusión}

Existe un incremento del uso de resinas compuestas como alternativa de tratamiento frente a lesiones cariosas, traumatismos, desgastes patológicos y otros tipos de lesiones, ya sea de forma directa, semi indirecta o indirecta; estos materiales tienen la desventaja de no tener adherencia por si sola a la estructura dental, necesitando de los sistemas adhesivos ${ }^{17,18}$. Siendo esta una de las razones de los constantes estudios de la interfaz dentina y/o esmalte- resina ${ }^{19}$. La evaluación de fuerzas de los sistemas adhesivos se puede realizar a través de fuerzas de cizalla o a través de fuerzas de tensión empleando el test de microtensión, este último resulta ser mejor técnica ya que permite observar fallas en valores elevados a $20 \mathrm{MPa}$ a diferencia del cizallamiento ${ }^{18}$.

El presente estudio experimental in vitro se realizó con el propósito de evaluar la resistencia del interfaz dentina resina mediante la utilización de agentes de unión como Etch and Rinse de tres pasos (Optibond FL) y dos pasos (Adper single Bond 2) y del adhesivo Multimodal sin grabado (Single Bond Universal). Para tal efecto se confeccionó especímenes acorde con las especificaciones y en base a estudios preliminares como el de Araya ${ }^{19}$, respetando la estandarización de la unidad de estudio en la presente investigación, donde fueron en total 135 especímenes. La confiabilidad de las mediciones fueron garantizadas por el uso de del microtensiómetro Microtensile Tester $\left(\mathrm{BISCO}^{\circ}\right.$ ) para medir las fuerzas de los sistemas adhesivos mediante el ensayo del test de microtensión. Para el mejor control de las variables se confeccionó especímenes utilizando un solo tipo de resina compuesta, que en este caso fue Filtek Z350 XT (3M). También se realizaron cortes mediante máquinas automatizadas y aplicando el mismo protocolo para cada espécimen.

Los datos obtenidos fueron procesados y analizados por un especialista en Bioestadística, el cual no conocía a qué grupos pertenecían los datos recolectados, lo que mejora la objetividad de los resultados.

Los hallazgos muestran que a mayor tiempo de aplicación mayor resistencia adhesiva, estos hallazgos son similares a algunos estudios como el de Lafuente ${ }^{4}$, quien menciona que dejar al adhesivo $30 \mathrm{~s}$ en dentina antes de fotocurar incrementa la fuerza adhesiva ${ }^{15}$, similar resultado obtuvieron De Carvalho et al ${ }^{20}$, Reis et al. ${ }^{3}$ concluyendo que los tiempos de aplicación prolongados pueden aumentar la resistencia adhesiva resina-dentina, en relación directa a la volatilización del solvente.

De acuerdo a la teoría, uno de los parámetros para el éxito en las restauraciones adhesivas a nivel de dentina, viene a ser la forma de aplicación del adhesivo ${ }^{5}$, no todos los fabricantes sugieren que deben ser aplicados efectuando una frotación ${ }^{5}$; son los estudios realizados en forma privada o alejado de las casas comerciales, como el de Amaral et al. ${ }^{21,22}$ que evidencian que una aplicación con frotación incrementa la fuerza adhesiva.

El grupo de adhesivos Etch and Rinse de tres pasos, son los adhesivos más antiguos y con mayores estudios de resistencia adhesiva, demostrando en todos ellos un alto nivel ${ }^{23}$, en el presente estudio, coincidentemente, con 
Tabla 5. Comparación de la resistencia adhesiva dentina-resina entre los diferentes sistemas adhesivos

\begin{tabular}{|c|c|c|c|c|c|c|}
\hline \multirow{2}{*}{ Frotación } & \multirow{2}{*}{ Adhesivos } & \multirow{2}{*}{$\begin{array}{l}\text { Diferencia de } \\
\text { medias (I-J) }\end{array}$} & \multirow{2}{*}{ Error estándar } & \multirow{2}{*}{ Valor $\mathrm{p}^{+}$} & \multicolumn{2}{|c|}{ IC $95 \%$} \\
\hline & & & & & Límite inferior & Límite superior \\
\hline \multirow{3}{*}{ Por 10 segundos } & A vs B & 11,76000 & 1,72684 & $<0,001^{*}$ & 7,5646 & 15,9554 \\
\hline & A vs C & 11,41867 & 1,72684 & $<0,001^{*}$ & 7,2233 & 15,6140 \\
\hline & B vs C &,- 34133 & 1,72684 & 0,979 & $-4,5367$ & 3,8540 \\
\hline \multirow{3}{*}{ Por 20 segundos } & A vs B & 18,03733 & 1,90004 & $<0,001^{*}$ & 13,4212 & 22,6535 \\
\hline & A vs C & 14,93600 & 1,90004 & $<0,001^{*}$ & 10,3199 & 19,5521 \\
\hline & B vs C & $-3,10133$ & 1,90004 & 0,244 & $-7,7175$ & 1,5148 \\
\hline \multirow{3}{*}{ Por 30 segundos } & A vs B & 20,98400 & 2,97355 & $<0,001^{*}$ & 13,7598 & 28,2082 \\
\hline & A vs C & 14,04667 & 2,97355 & $<0,001^{*}$ & 6,8225 & 21,2709 \\
\hline & B vs C & $-6,93733$ & 2,97355 & 0,062 & $-14,1615$ & 0,2869 \\
\hline
\end{tabular}

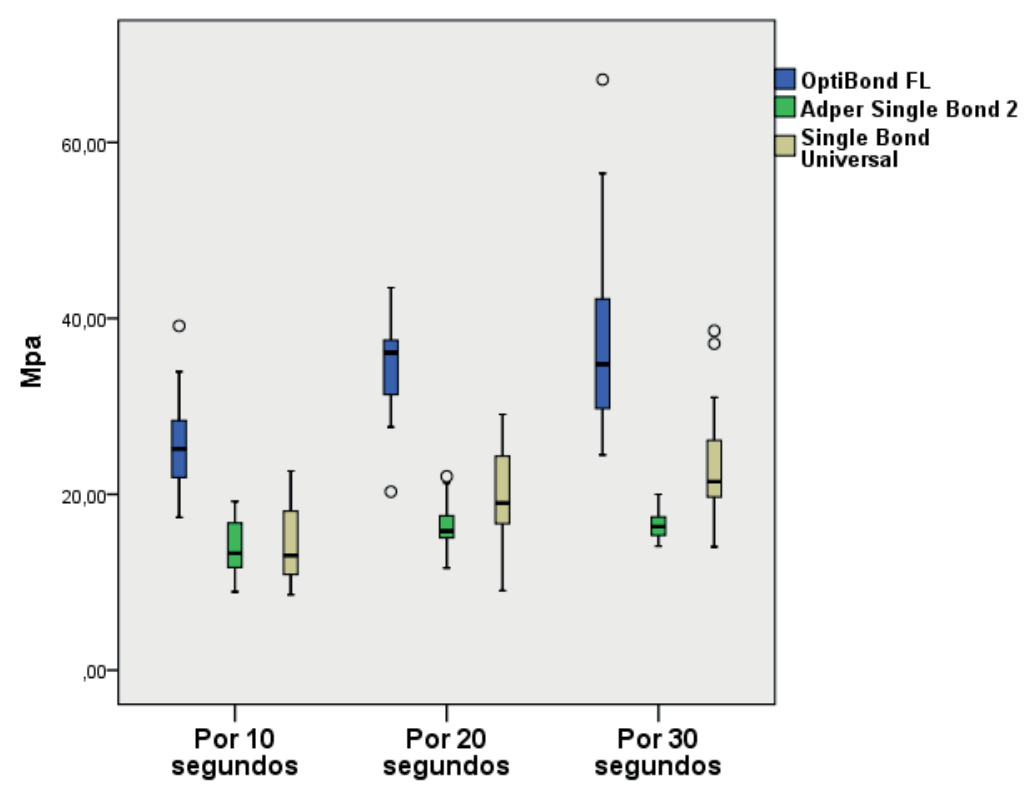

Figura 5. Distribución de valores de resistencia adhesiva entre los diferentes sistemas adhesivos por grupos de frotación

el adhesivo Optibond FL se encontró niveles superiores frente al Etch and Rinse de dos pasos (Adper Single Bond 2) y frente al adhesivo Multimodal (Single bond Universal), ello debido a la utilización del "primer" y bonding por separado, favoreciendo la infiltración efectiva en primera instancia solo del "primer" para luego aplicar el bonding que viene a ser una capa hidrófoba que actúa protegiendo a la capa inferior hidrófila ("primer")

En los grupos de sistemas adhesivos Etch and Rinse de dos pasos como el que se empleó en la presente investigación (Adper Single Bond 2), presentan el "primer" y el bonding mezclados en un solo frasco, incorporando monómeros de diferente peso molecular, afinidad opuesta al agua y con objetivos diferentes ${ }^{19}$, lo que conlleva a errores por parte del profesional; como no agitar el frasco para la integración de ambos, ello podría ser una de las causas de la no obtención de valores de resistencia adhesiva similares al adhesivo Etch and Rinse de tres pasos.
Dentro de los beneficios de los adhesivos Multifuncionales es que presentan la molécula bifuncional vinil silano asimismo de monómeros funcionales que se unen a la hidroxiapatita brindando una unión química y micromecánica ${ }^{13}$, además de la posibilidad de ser empleado en forma de autograbado a nivel de dentina (para la no activación de las metaloproteinasas), estas ventajas asociadas al nivel de resistencia adhesiva mayores, en sus tres tiempos de frotación, encontradas en el presente estudio para el adhesivo Multimodal single Bond Universal, frente al Adhesivo Etch and Rinse de dos pasos Adper Single Bond 2, conducirían a tener una mayor demanda de los adhesivos Multimodales.

Los valores de resistencia adhesiva dentina-resina obtenidos para los tres adhesivos ameritan de posteriores estudios en el tiempo para analizar si dichos valores incrementan, disminuyen o se mantienen, sobre todo en el sistema adhesivo Etch and Rinse de tres pasos: Optibond $F L$, donde se observa valores elevados y se emplea el ácido fosfórico estimulando la liberación de metaloproteinasas. 
Como limitación para el desarrollo de la presente investigación, se menciona la falta del análisis del patrón de fractura para evidenciar si el fallo fue por la resina, el adhesivo o mixto, debido a la existencia de muy pocos centros de investigación que cuenten con los equipos necesarios, por lo que podría ser motivo de futuras investigaciones.

Se debe tener en cuenta que para el éxito de los sistemas adhesivos en dentina se deben de considerar varios factores como son el acondicionamiento ácido, tiempo de lavado, forma de secado, humedad de la dentina, aplicación activa del adhesivo (frotación), tiempo de aplicación del adhesivo, evaporización del solvente, fotocurado de adhesivo y por último el agregado de una capa hidrófoba de adhesivo para minimizar la hidrólisis del mismo ${ }^{15}$, logrando adecuados niveles de resistencia adhesiva y estos se puedan conservar en el tiempo incrementando la longevidad de la interfaz adhesiva.

\section{Agradecimiento}

$\mathrm{Al}$ Dr. Miguel Saravia por la viabilidad del equipo $M i$ crotensile Tester (BISCO®).

\section{Referencias bibliográficas}

1. Ramos G, Ramírez N, Medina R. Adhesión convencional en dentina, dificultades y avances en la técnica. Rev Fac Odontol Univ Antioq [Internet]. 2015;26(2):468486. Disponible en: http://www.scielo.org.co/pdf/rfoua/ v26n2/v26n2a13.pdf

2. Pignata Volpe S, Vola J. Importancia de la Interfaz Dentina-Adhesivo en la Longevidad de las Restauraciones Adheridas. RODYB [Internet]. 2015;4(1):34-42. Disponible en: https://www.colibri.udelar.edu.uy/jspui/handle/123456789/11113

3. Reis A, De Carvalho P, Clovis L et al. Effect of prolonged application times on the durability of resin-dentin bonds. Dent Mater J. 2008;24(1):639-644.

4. Lafuente D. Aumento en la fuerza de adhesión a dentina con una capa extra de adhesivo dentinal. J Dent [Internet]. 2010;12(1):22-25. Disponible en: https://revistas. ucr.ac.cr/index.php/Odontos/article/view/4778/4588

5. Silva J, Otto H, Rodriguez RR. Principios de Adhesión dentinaria. ODOUS científica. [Internet]. 2014;3 (2): Disponible en: servicio.bc.uc.edu.ve/odontologia/revista/v3n2/3-2-3.pdf.

6. Garrofé A, Martucci D, Picca M. Adhesión a tejidos dentarios. Revista de la Facultad de Odontología (Universidad de Buenos Aires) .[Internet]. 2014;29 (67): 5-13. Disponible en: od.odontologia.uba.ar/revista/pdf/ revvol29-67.pdf

7. Rosa WL, Piva E, Silva AF. Bond strength of universal adhesives: A systematic review and meta-analysis. J Dent. 2015;43(7):765-76.

8. Bader M, Ibáñez M. Evaluación de la interfase adhesiva obtenida en restauraciones de resina compuesta realizadas con un sistema adhesivo universal utilizado con y sin grabado ácido previo. Rev Clin Periodoncia Implantol Rehabil Oral. [Internet]. 2014;7(3):115-122. Doi: https://doi.org/10.1016/j.piro.2014.09.005
9. Verastegui GA. Análisis in vitro de la resistencia adhesiva a la tracción entre dos sistemas adhesivos, uno de 5o generación (adper single bond de $3 \mathrm{~m}$ ) y de 6o generación (adper prompt l-pop de $3 \mathrm{~m}$ ), en molares premanentes. [Tesis de Bachiller].Tacna-Perú: Facultad de ciencias de la salud, Universidad Nacional Jorge Basadre Grohmann. 2016. $31 \mathrm{p}$

10. Tello R, Huertas G, Aguirre C, Cáceres J, Bragagnini P. Rehabilitación Oral Adhesiva con sistemas adhesivos Etch and Rinse y Self Etch. Revista Odontos. 2018;50:70-78.

11. Mandri MN, Aguirre A, Zamudio ME. Sistemas adhesivos en Odontología Restauradora. Revista Odontoestomatología. [Internet]. 2015; 17(26):50-56. Disponible en: www.scielo.edu.uy/pdf/ode/v17n26/v17n26a06.pdf

12. Kose C, Andrade PE, Serrano AP, Tay Y, Reis A, Loguercio $\mathrm{AD}$, et al.. Apliacação de um novo sistema adesivo universal: relato de caso. Rev Assoc Paul Cir Dent [Internet]. 2013;67(3):202-206. Disponible en: revodonto. bvsalud.org/scielo.php.52762013000300006.

13. Arinell Dib; Pereira F, Prado S, Rabello B. Sistemas adhesivos atuais. Rev Bras Odontol. [Internet].2016;73(3):242-246. Disponible en: http://revodonto.bvsalud.org/scielo.php?pi$\mathrm{d}=$ S0034-72722016000300012\&script=sci_arttext.

14. Vélez EM, Zulay CM, Álvarez DP. Operatoria dental y endodoncia. Protección de la interfaz resina-dentina mediante inhibidores de las enzimas colagenolíticas. Rev Asoc Odontol Argent. 2018;106:70-76.

15. Hidalgo RC. Reacción de la dentina a los sistemas adhesivos resinosos: aspectos biológicos relacionados y biodegradación de la capa híbrida. Rev Estomatol Hered. [Internet]. 2018;18(1). Disponible en: http://www.upch. edu.pe/vrinve/dugic/revistas/index.php/REH/article/ viewFile/1855/1864

16. Cardoso M, Neves A, Mine et al. Current aspects on bonding effectiveness and stability in adhesive dentistry. Aust Dent J. [Internet]. 2011;56(1):31-44. Disponible en: www.ncbi.nlm.nih.gov/pubmed/21564114

17. Halim N, Nasser HF, Tebechrani CF, Drumond H, Guerino M. Sistemas adesivos - classificaçáo. Full dent. sci. [Internet]. 2014;5(20):641-6. Disponible en: www.researchgate.net/profile/Halim_Filho/publication/272476475_Adhesives_systems_classification/ links/5666ef2508ae34c89a01b5b9/Adhesives-systems-classification.pdf

18. Sano H, Shono T, Sonoda H. Relationship between surface area for adhesion and tensile bond strengthevaluation of a micro-tensile bond test. Dent Mater J.1994;10(1):236-240.

19. Araya M. Análisis Comparativo In Vitro de la Resistencia Adhesiva de las Restauraciones de Resina Compuesta Utilizando el adhesivo Peak Universal Bond con y sin grabado acido previo de superficie. [Tesis de bachiller]. [Lima]: Facultad de Odontología, Departamento de Restauradora- Área de Biomateriales, Universidad de Chile.2014. 31p.

20. De Carvalho P, Dourado A, Clovis L et al. Effect of Prolonged Application Times on Resin-Dentin Bond Strengths. J Adhes Dent. 2005;7(1):143-149.

21. Do Amaral R, Stanislawczuk R, Zander-Grande C et al. Active application improves the bonding performance 
of self-etch adhesives to dentin. J Dent. [Internet]. 2009;37(1):82-90. Disponible en: https://www.ncbi. nlm.nih.gov/pubmed/19013000

22. Do Amaral R, Stanislawczuk R, Zander-Grande C et al. Bond Strength and Quality of the Hybrid Layer of Onestep Self-etch Adhesives Applied with Agitation on Dentin. Operative Dentistry. [Internet]. 2010;35(2):211-
219. Disponible en: https://www.ncbi.nlm.nih.gov/ pubmed/20420065.

23. Silva M, Carneiro K, Lobato M, et al. Adhesive systems: important aspects to their composition and clinical use. J Appl Oral Sci [Internet]. 2010;18(3):207-214. Disponible en: www.ncbi.nlm.nih.gov/pubmed/20856995. 
